\title{
Etude de la pénétration et de la stabilité de la pimaricine dans les fromages à pâte molle
}

\author{
par \\ J.C. GRIPON et J.L. BERGERE \\ Station Centrale de Recherches Laitières et de Technologie des Produits Animaux, \\ I.N.R.A., Jouy-en-Josas (Yvelines)
}

\section{INTRODUCTION}

La pimaricine est un antibiotique produit par Streptomyces natalensis actif à de faibles concentrations sur les moisissures et les levures. De par sa structure chimique cet antibiotique appartient comme la nistatine au groupe des antibiotiques polyènes. Il est actif et stable entre $\mathrm{pH} 4,5$ et 7,0 et sa solubilité dans l'eau est faible (0,005 p. 100).

L'utilisation de la pimaricine a été préconisée afin de résoudre le problème difficile de l'entretien des surfaces de fromages à croûte non moisie surtout lorsqu'ils ne sont pas enrobés. Cet entretien implique en effet des frottages et brossages réguliers des surfaces qui exigent une main-d'œuvre importante et dont l'efficacité n'est pas toujours satisfaisante. La pimaricine a été expérimentée d'abord en Hollande sur des fromages de Gouda ; depuis son usage s'est officialisé et répandu dans d'autres pays. La pimaricine est actuellement autorisée en France sous certaines conditions dans les fromages à pâte pressée. L'intérêt de cet antibiotique pour d'autres types de fromages à croûte non moisie est évident. Parmi tous les problèmes que pose l'utilisation d'un antibiotique sur la croûte des fromages ceux de sa stabilité et de sa pénétration sont de première importance. Ces deux points ont déjà été examinés dans le cas du Gouda (Mol, 1966), mais nous manquons d'informations pour les pâtes molles à croûte non moisie. Le présent travail a eu pour objet d'étudier la stabilité et la pénétration de la pimaricine dans ce type de caillé.

\section{TECHNIQUES ET METHODES}

\section{1) Préparation et traitement des caillés}

a) Fabrication et affinage des caillés

Trois types de caillé ont été choisis représentant trois catégories de fromages : fromages à coagulation présure type Pont-l'Evêque, 
fromages à coagulation lactique type Munster et enfin fromages type Bleu de Bresse afin d'observer l'influence éventuelle de la pimaricine sur le développement interne des moisissures. Ces fromages ont été préparés au laboratoire.

Les trois types de caillé étaient affinés à $10^{\circ} \mathrm{C}$ et $95 \mathrm{p} .100$ d'humidité relative. Après 15 jours les «Bleu de Bresse " étaient enveloppés dans du papier d'aluminium sans subir de frottage ou de nettoyage. Par contre les "Pont l'Evêque » et les « Munster » étaient frottés à l'aide d'un chiffon imprégné d'une suspension de Brevibacterium linens, cette opération étant effectuée deux fois pendant la première semaine, puis une fois par semaine pendant 1 mois. Le but de cette opération était de faciliter l'installation d'une flore bactérienne de surface.

\section{b) Application de la pimaricine}

La pimaricine utilisée était une préparation commerciale ( " Delvocid ") consistant en une suspension aqueuse à 5 p. 100 de pimaricine ou des dilutions en eau distillée de cette préparation.

Pour les essais concernant la stabilité de la pimaricine à forte concentration effectués sur les « Munster ", $0,75 \mathrm{ml}$ d'une suspension de pimaricine à 5 p. 100 était déposé sur chacune des deux faces de chaque fromage (soit au total $1,5 \mathrm{ml}$ par fromage) et étalé à l'aide d'un pinceau imprégné de la même suspension. Ces opérations ont été effectuées 5 ou 21 j après la fabrication. Pour les essais concernant la pénétration de la pimaricine une suspension aqueuse de pimaricine à 0,1 ou 1 p. 100 était appliquée à la surface des fromages à l'aide d'un pinceau imbibé de la suspension de pimaricine. Les volumes des suspensions appliquées ainsi par fromage ont été de 1,3 ml pour les "Pont-l'Evêque » et de 1,9 ml pour les « Munster ". Compte tenu de la surface des fromages, les quantités de pimaricine se trouvant à la surface après traitement avec la suspension à $0,1 \mathrm{p} .100$ était de $3,3 \mu \mathrm{g} / \mathrm{cm}^{2}$ pour les "Pont-l'Evêque » et de $6 \mu \mathrm{g} / \mathrm{cm}^{2}$ pour les " Munster ». Le traitement était effectué après passage en saumure et un premier frottage des fromages. En outre 0,025 p. 100 de pimaricine a été ajouté à la suspension bactérienne utilisée pour les frottages ultérieurs. Cette addition avait pour objectif d'arrêter le développement des moisissures installées dans les anfractuosités des caillés non atteintes par la pimaricine lors du traitement au pinceau et de compenser une élimination partielle de la pimaricine au cours des frottages.

Les "Bleu de Bresse » ont été traités soit avant, soit après piquage sans subir de frottages ultérieurs.

La répartition des 7 lots expérimentaux composés chacun de 12 fromages était la suivante :

- "Pont l'Evêque " traités avec une suspension à 0,1 p. 100 de pimaricine + frottages $(0,025 \mathrm{p} .100$ de pimaricine $)$. 
- "Pont l'Evêque » traités avec une suspension à 1 p. 100 de pimaricine + frottages $(0,025 \mathrm{p} .100$ de pimaricine $)$.

- "Munster » traités avec une suspension à 0,1 p. 100 de pimaricine + frottages $(0,025$ p. 100 de pimaricine $)$.

- "Munster " traités avec une suspension à 1 p. 100 de pimaricine + frottages $(0,025$ p. 100 de pimaricine $)$.

— "Bleu de Bresse » traités avec une suspension à 1 p. 100 de pimaricine avant piquage.

- "Bleu de Bresse " traités avec une suspension à 0,1 p. 100 de pimaricine après piquage.

- "Bleu de Bresse " traités avec une suspension à 1 p. 100 de pimaricine après piquage.

Dix fromages témoins furent conservés pour chaque type de fromage.

\section{2) Dosage de la pimaricine}

1. PRINCIPE ET DESCRIPTION DE LA MÉTHODE

La pimaricine est dosée par la méthode de diffusion en gélose. Le germe test est une souche de Saccharomyces cerevisiae (ATCC 9763) entretenue par repiquage sur une gélose au malt.

a) Préparation des plaques

Le milieu de culture est le milieu Wiffen tamponné aont la composition est la suivante :

$\begin{array}{ll}\text { Extrait de levure Difco } & 2,5 \mathrm{~g} \\ \mathrm{KH}_{3} \mathrm{PO}_{4} & 6,8 \mathrm{~g} \\ \text { Soude } & 0,6 \mathrm{~g} \\ \text { Gélose } & 15 \mathrm{~g} \\ \text { Eau distillée } & 1000 \mathrm{ml} \\ \text { pH final après stérilisation } & 6,5\end{array}$

Ce milieu est filtré à chaud et son pH est ajusté à 6,6 avec de la soude. Après stérilisation par autoclavage à $110^{\circ} \mathrm{C}$ pendant $30 \mathrm{mn}$ une solution de glucose à 50 p. 100 stérilisée par filtration est ajoutée au milieu à raison de $2 \mathrm{ml}$ pour $100 \mathrm{ml}$.

$\mathrm{Au}$ moment de la préparation des plaques de milieu gélosé le milieu préalablement fondu puis refroidi à $45^{\circ} \mathrm{C}$ est ensemencé à 1 p. 100 avec une préculture de $48 \mathrm{~h}$ du germe-test effectuée à $26^{\circ} \mathrm{C}$ dans un milieu liquide contenant $125 \mathrm{~g} / 1$ d'extrait de malt préalablement filtré.

Le milieu gélosé ensemencé est coulé sur des plaques de verre carrées de $16 \mathrm{~cm}$ de côté stérilisées au four Pasteur préalablement chauffées à $45^{\circ} \mathrm{C}$ et placées sur une surface plane. Chaque plaque reçoit $20 \mathrm{ml}$ de milieu.

b) Dépôt des échantillons

Les échantillons à doser se présentent soit sous forme de disques de fromage, soit sous forme d'extraits méthanoliques de fromage. 
Les disques de fromage sont déposés directement à la surface de la gélose. Dans le cas des extraits méthanoliques ou des solutions étalons des anneaux de vanadium de $6 \mathrm{~mm}$ de diamètre intérieur et $8 \mathrm{~mm}$ de diamètre extérieur sont posés à la surface de la gélose. $0,05 \mathrm{ml}$ d'extrait ou de solution étalon est introduit à l'intérieur de chaque anneau à l'aide d'un tube capillaire.

\section{c) Incubation et lecture}

Pour éviter la dessiccation de la gélose les plaques sont préalablement placées à l'intérieur de boîtes métalliques. Ensuite elles sont d'abord maintenues pendant $2 \mathrm{~h}$ à $4^{\circ} \mathrm{C}$ puis incubées pendant $18 \mathrm{~h}$ à $30^{\circ} \mathrm{C}$. Après incubation le diamètre de la zone d'inhibition obtenue autour de chaque échantillon est mesuré. Le dépôt des échantillons et l'incubation des plaques sont effectués dans des conditions non aseptiques.

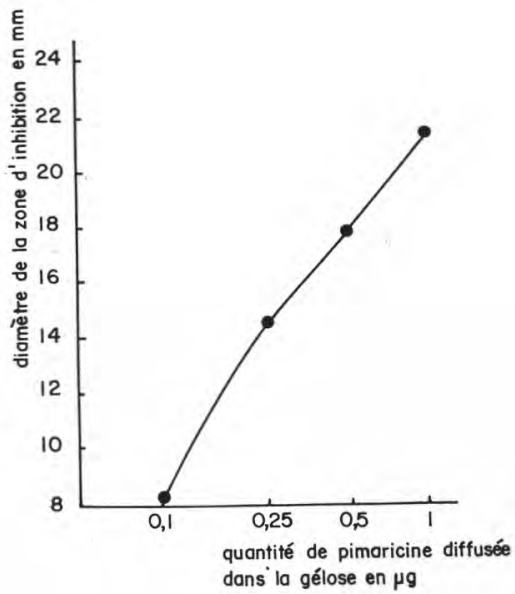

fig. 1

Courbe étalon de la pimaricine

d) Etalonnage

Il a été vérifié que les zones d'inhibition obtenues étaient bien dues à la présence de pimaricine. L'addition au milieu gélosé d'une enzyme détruisant la pimaricine (la pimaricinase) et des dosages effectués sur des fromages témoins non traités ont permis ce contrôle.

Une courbe étalon est établie à l'aide de solutions de pimaricine en méthanol a 50 p. 100. Les concentrations des solutions utilisées sont de $2,5,10$ et $20 \mu \mathrm{g} / \mathrm{ml}$.

Une relation linéaire entre le diamètre de la zone d'inhibition et le logarithme de la concentration en pimaricine est obtenue pour 
des concentrations comprises entre 5 et $20 \mu \mathrm{g} / \mathrm{ml}$. Il ne se forme pas de zone d'inhibition pour des concentrations de pimaricine inférieu. res à $2 \mu \mathrm{g} / \mathrm{ml}$. Pour tenir compte du fait que dans les disques de fromage on ignore a priori si toute la pimaricine présente dans le disque diffuse dans la gélose, on a gradué la courbe étalon en quantité de pimaricine ayant diffusé dans la gélose. (Dans le cas des solutions étalons toute la pimaricine diffuse dans la gélose).

Pour les dosages effectués par extraction méthanolique, chaque extrait de fromage était comparé à une courbe étalon établie sur la même plaque de gélose que l'extrait. Pour les dosages par la méthode des disques une seule courbe étalon a été utilisée pour tous les dosages (fig. 1). Elle a été établie en prenant les valeurs moyennes des diamètres des zones d'inhibition obtenues sur 10 gammes étalons avec des solutions de concentration 2, 5, 10 et $20 \mu \mathrm{g} / \mathrm{ml}$. (Les quantités de pimaricine ayant diffusé dans la gélose étaient respectivement de $0,1,0,25,0,5$ et $1 \mu \mathrm{g}$ ). Les courbes obtenues d'une plaque à l'autre et d'un jour à l'autre n'étant pas exactement superposables, l'erreur relative sur les quantités de pimaricine ayant diffusé dans la gélose, déterminées à partir de cette courbe moyenne, est au maximum de 25 p. 100.

\section{Méthodes DES DISQues}

\section{a) Préparation des disques}

Des tranches de $1 \mathrm{~mm}$ d'épaisseur sont découpées parallèlement à la surface du fromage à l'aide d'un couteau. Un disque de $8 \mathrm{~mm}$ de diamètre (soit $0,5 \mathrm{~cm}^{2}$ ) est découpé dans chaque tranche à l'aide d'un perce-bouchon. Pour faciliter le découpage des disques à partir de fromages en cours d'affinage ceux-ci sont maintenus pendant $2 \mathrm{~h}$ à $-25^{\circ} \mathrm{C}$ avant découpage.

\section{b) Dépôt des disques}

La face supérieure des disques est placée directement en contact avec la gélose. Pour le premier mm deux disques ont été préparés, l'un étant placé la face supérieure en contact avec la gélose, l'autre étant placé la face inférieure en contact avec la gélose.

$\mathrm{Au}$ cours de l'incubation, les disques de fromage provenant de fromages dont l'affinage est déjà avancé ont tendance à s'étaler et donc à recouvrir la zone d'inhibition. Pour éviter cet étalement les disques sont placés à l'intérieur d'un anneau de verre de $8 \mathrm{~mm}$ de diamètre intérieur et de $11 \mathrm{~mm}$ de diamètre extérieur. Dans ce cas la sensibilité de la méthode se trouve légèrement diminuée (elle est alors de $0,15 \mu \mathrm{g} /$ disque) puisque la lecture ne s'effectue plus que sur des zones de diamètre supérieur à $11 \mathrm{~mm}$.

En outre, on a observé que des anneaux opalescents venaient se superposer à la zone d'inhibition. A l'examen au microscope l'anneau de plus petit diamètre se présentait comme un précipité alors que le plus externe se révélait être une zone d'activation des levures. La 
présence et l'interprétation de ces anneaux furent confirmés par l'examen de plaques additionnées de pimaricinase ou de plaques préparées à l'aide de fromages témoins. La présence de ces anneaux n'a pas gêné la détermination du diamètre des zones d'inhibition.

\section{c) Expression des résultats}

Les analyses ont été effectuées chaque semaine à partir du lendemain du traitement pendant 4 semaines.

Les résultats ont été exprimés sous forme d'histogramme représentant les quantités de pimaricine détectables en fonction de la profondeur du disque (exprimées en $\mu \mathrm{g}$ par $\mathrm{cm}^{2}$ de section de fromage analysé).

\section{$3^{\circ}$ DOSAGE APRÈs EXTRACTION PAR LE MÉTHANOL}

Une portion de fromage est coupée en petits morceaux que l'on soumet à trois extractions successives par du méthanol à 100 p. 100 Pour chaque extraction les morceaux de fromage sont mis en suspension dans le méthanol et agités pendant $30 \mathrm{mn}$ à la température ordinaire et à l'abri de la lumière. Les extraits sont dilués par addition d'un même volume d'eau distillée. Si la concentration de l'extrait est trop élevée, l'extrait est à nouveau dilué avec du méthanol à 50 p. 100 . Ce sont ces solutions en méthanol à 50 p. 100 qui sont utilisées pour le dosage de la pimaricine.

Dans le cas des caillés "Munster » traités avec une suspension de pimaricine à $5 \mathrm{p}$. 100 , un secteur circulaire pesant environ $10 \mathrm{~g}$ était prélevé dans toute la hauteur du caillé découpé en petits morceaux et extrait successivement avec 100,25 et $25 \mathrm{ml}$ de méthanol à 100 p. 100 . Dans ces conditions le seuil de sensibilité du dosage était de $40 \mu \mathrm{g} / \mathrm{g}$ de fromage.

Dans le cas des caillés traités avec la suspension de pimaricine à $0,1 \mathrm{p} .100$ un morceau de $5 \mathrm{~mm}$ d'épaisseur et $12 \mathrm{~cm}^{2}$ de surface (soit environ $6 \mathrm{~g}$ de caillé) était prélevé à la surface du fromage, découpé et extrait successivement avec 15,5 et $3 \mathrm{ml}$ de méthanol à 100 p. 100. La sensibilité du dosage était de $10 \mu \mathrm{g} / \mathrm{g}$ de fromage ou bien $5 \mu \mathrm{g} / \mathrm{cm}^{2}$ de surface du fromage.

Les caillés traités avec la suspension de pimaricine à 5 p. 100 ont été analysés 2 et $15 \mathrm{j}$ après le premier traitement et $21 \mathrm{j}$ après le second traitement. Ceux traités avec la suspension à $0,1 \mathrm{p} .100$ ont été analysés au cours de la $5^{\mathrm{me}}$ semaine d'affinage pour les trois types de caillé.

\section{RESULTATS ET DISCUSSION}

\section{1) Stabilité de la pimaricine}

La pimaricine peut être inactivée par certains facteurs physicochimiques (Struyk et al., 1957 ; Clark et al., 1964) tels que la lumière 
ou l'oxydation et par une enzyme produite par certains microorganismes. Il était donc nécessaire de tester sa stabilité dans les caillés expérimentaux.

\section{a) Stabilité à forte concentration}

La teneur en pimaricine des caillés « Munster » traités avec une suspension de pimaricine à 5 p. 100 a été déterminée par extraction au méthanol. Les résultats donnés dans le tableau 1 font apparaître que la quantité de pimaricine retrouvée demeure constante quelle que soit la date du traitement et de l'analyse. Cette quantité représente environ 65 p. 100 de la quantité initiale.

TABLEAU 1. - Stabilité de la pimaricine dans les caillés " Munster »

\begin{tabular}{|c|c|c|c|c|}
\hline \multirow[t]{2}{*}{1} & \multicolumn{2}{|c|}{$\begin{array}{c}\text { Traitement après } 5 \mathrm{j} \\
\text { de fabrication }\end{array}$} & \multicolumn{2}{|c|}{$\begin{array}{c}\text { Traitement après } 21 \mathrm{j} \\
\text { de fabrication }\end{array}$} \\
\hline & $\begin{array}{l}\text { Analyse } 2 \mathrm{j} \\
\text { après le } \\
\text { traitement }\end{array}$ & $\begin{array}{l}\text { Analyse } 25 \mathrm{j} \\
\text { après le } \\
\text { traitement }\end{array}$ & \multicolumn{2}{|c|}{$\begin{array}{l}\text { Analyse } 21 \mathrm{j} \text { après } \\
\text { le traitement }\end{array}$} \\
\hline Quantité retrouvée & 57700 & 54000 & 58400 & 57200 \\
\hline$\mu \mathrm{g} /$ fromage & 52700 & 50300 & & \\
\hline $\begin{array}{l}\text { p. } 100 \text { de la quantité } \\
\text { appliquée }\end{array}$ & $\begin{array}{l}70 \\
64\end{array}$ & $\begin{array}{l}66 \\
61\end{array}$ & 71 & 69 \\
\hline \multicolumn{5}{|c|}{ Quantité appliquée par fromage : $82500 \mu \mathrm{g}$} \\
\hline
\end{tabular}

La différence de 35 p. 100 ne peut s'expliquer par une destruction rapide de la pimaricine puisque celle-ci devrait avoir lieu dans les deux premiers jours sans qu'il y ait de destruction ultérieure. La différence observée doit donc être attribuée à la méthode d'extraction de la pimaricine qui apparaît comme incomplète. Dans ces conditions le fait qu'il n'y ait pas de variation de la quantité retrouvée, que le dosage soit effectué 2,15 ou $21 \mathrm{j}$ après le traitement est un argument en faveur d'une très bonne stabilité de la pimaricine dans le fromage.

b) Stabilité à faible concentration

La précision des mesures dans l'essai précédent est insuffisante pour détecter l'inactivation d'une faible quantité de pimaricine. 
A titre de vérification des dosages ont été effectués sur les caillés des trois types étudiés traités avec une suspension de pimaricine à 0,1 p. 100 . Ces dosages ont été effectués par extraction au méthanol au cours de la $5^{\text {me }}$ semaine d'affinage. Les valeurs obtenues étaient à la limite de la sensibilité de la méthode soit $5 \mu \mathrm{g} / \mathrm{cm}^{2}$ de surface de caillé. La quantité initiale de pimaricine appliquée en surface ne peut être exprimée avec précision car les caillés ont subi, dans le cas du "Pont l'Evêque " et du " Munster ", des frottages à 0,025 p. 100 de pimaricine. Nous avons estimé que la quantité totale de pimaricine appliquée était comprise entre 3 et $6 \mu \mathrm{g} / \mathrm{cm}^{2}$ de surface de caillé pour les "Pont-l'Evêque » et 6 à $12 \mu \mathrm{g} / \mathrm{cm}^{2}$ de surface pour les "Munster ». Compte tenu de l'imprécision des données il est impossible de calculer le taux de pimaricine inactivée, mais, en tout état de cause, les quantités de pimaricine inactivées sont faibles.

Il apparaît donc que la pimaricine reste stable dans les caillés expérimentaux. La stabilité de la pimaricine dans le fromage est un phénomène qui a déjà été observé dans le cas des faibles doses applicables dans la pratique. Pour des fromages de Gouda enveloppés dans une émulsion plastique à $0,05 \mathrm{p}$. 100 de pimaricine, Mol (1966) a relevé la présence de pimaricine jusqu'à 10 semaines après le traitement. Von Lorch et al. (1968) signalent que la pimaricine peut être détectée sur des fromages à pâte molle jusqu'à 10 semaines après le traitement.

\section{TABLEAU 2}

Quantité de pimaricine détectée par $\mathrm{cm}^{2}$ de surface de fromage en fonction du temps

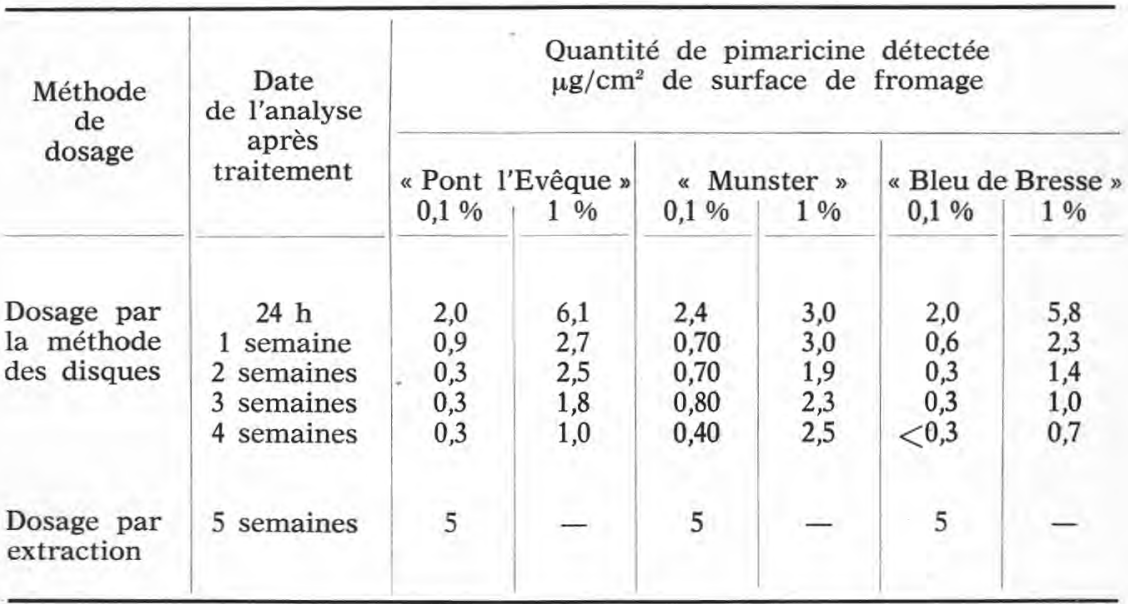

\section{2) Etude de la pénétration}

La méthode des disques a été utilisée afin de détecter et doser la présence de pimaricine à différentes profondeurs dans le caillé. La 


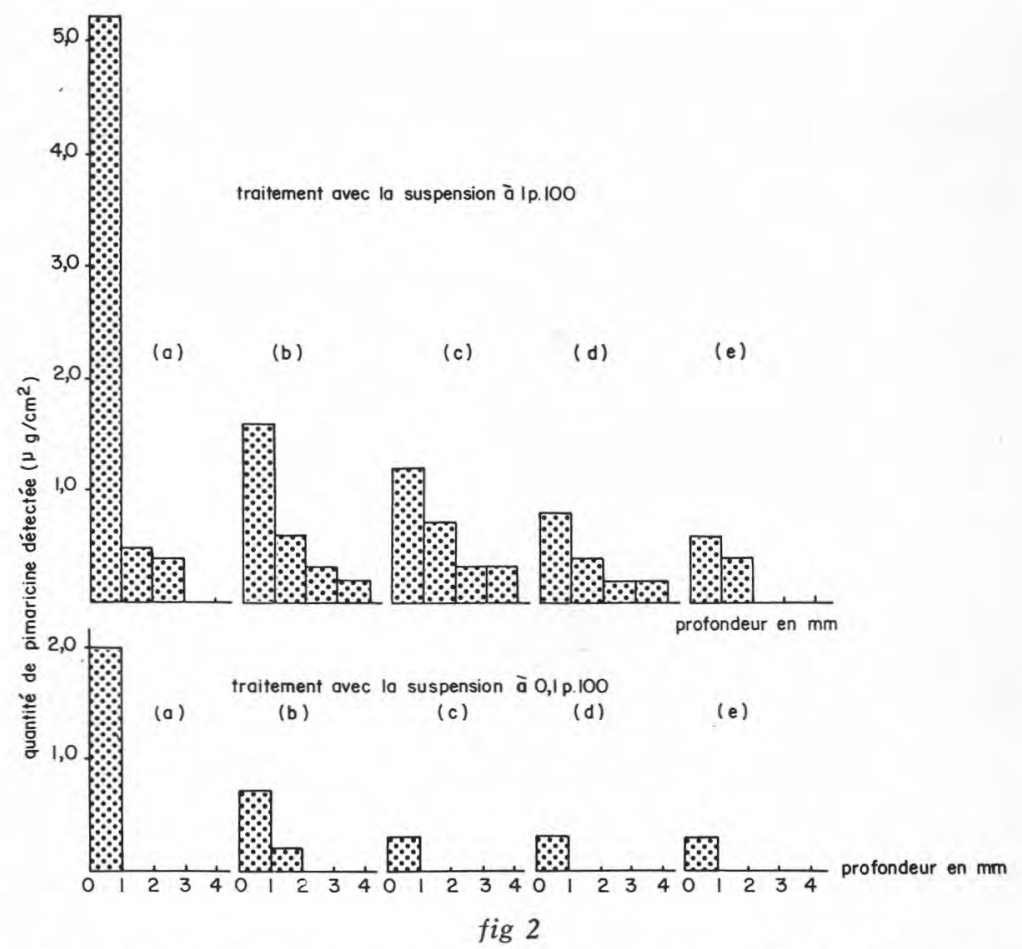

Pénétration de la pimaricine dans le « Pont l'Evêque » (Méthode des disques). Quantité de pimaricine détectée par $\mathrm{cm}^{2}$ à différentes profondeurs, $24 \mathrm{~h}(\mathrm{a})$, 1 semaine (b), 2 semaines (c), 3 semaines (d), 4 semaines (e) après traitement.

somme des quantités de pimaricine détectées dans les disques prélevés à différentes profondeurs est exprimée dans le tableau 2.

a) "Pont l'Evêque " (fig. 2)

- Traitement avec la suspension à 1 p. 100 : la quantité de pimaricine détectable décroît d'un disque à l'autre, il y a donc formation d'un gradient de concentration. La profondeur maximale de détection qui est de $4 \mathrm{~mm}$ est atteinte dès la $1^{\text {re }}$ semaine.

- Traitement avec la suspension à 0,1 p. 100 : au début, on observe une diminution progressive de la quantité de pimaricine détectable. La profondeur maximale de détection est de $2 \mathrm{~mm}$.

b) «Munster» (fig. 3)

- Traitement avec la suspension à 1 p. 100 : comme dans le cas du «Pont l'Evêque » on observe la formation d'un gradient de concentration en pimaricine. Au cours des 4 semaines de maturation la pente du gradient devient de plus en plus faible et le bilan de la pimaricine 


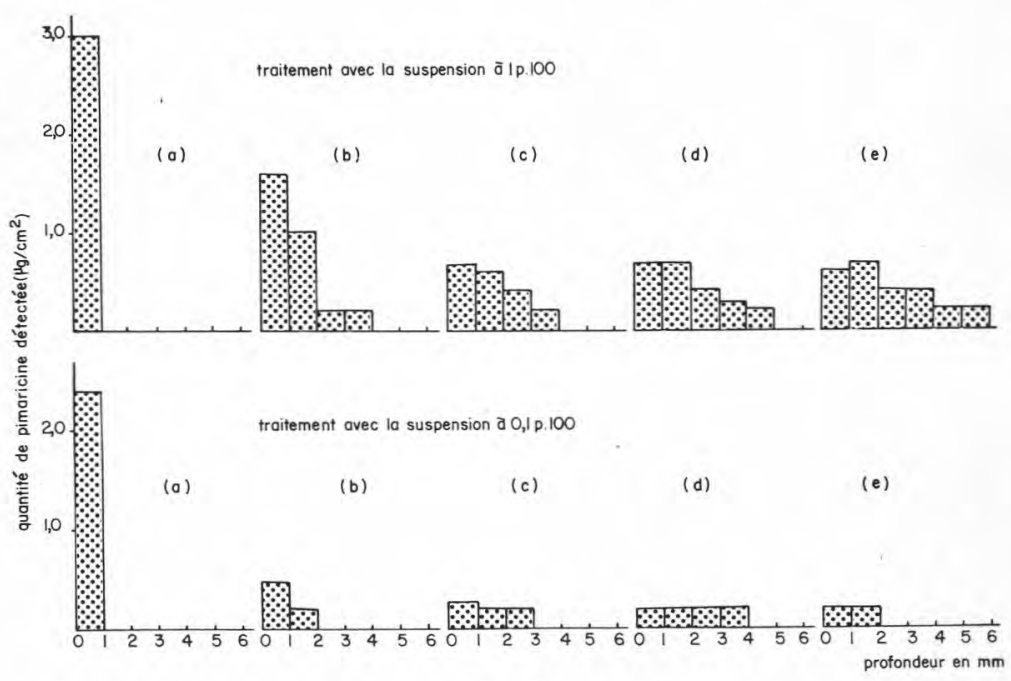

fig. 3

Pénétration de pimaricine dans le "Munster " (Méthode des disques). Quantité de pimaricine détectée par $\mathrm{cm}^{2}$ à différentes profondeurs, $24 \mathrm{~h}(\mathrm{a})$, 1 semaine (b), 2 semaines (c), 3 semaines (d), 4 semaines (e) après traitement.

détectable reste constant (tab. 2). La pénétration est détectée à une profondeur maximale de $5 \mathrm{~mm}$ au cours de la $4^{\text {me }}$ semaine.

- Traitement avec la suspension à 0,1 p. 100 : on observe également la formation d'un gradient mais le bilan diminue progressivement au cours de l'affinage. La profondeur maximale de détection $(4 \mathrm{~mm})$ est obtenue après 3 semaines d'affinage.

c) "Bleu de Bresse " (fig. 4)

- Traitement avec la suspension à $1 \mathrm{p} .100$ : la pimaricine n'est détectée à une profondeur supérieure à $2 \mathrm{~mm}$. On observe un gradient très rapide et une diminution importante du bilan (tab. 2).

- Traitement avec la suspension à 0,1 p. 100 : la pimaricine ne peut être détectée à une profondeur supérieure au premier $\mathrm{mm}$. La quantité détectée diminue très rapidement et après 4 semaines la pimaricine n'est plus détectable.

- L'observation à l'œil nu de sections de fromages n'a pas permis de remarquer de différences concernant le développement des moisissures internes entre les fromages témoins et les fromages traités à 0,1 ou 1 p. 100. 


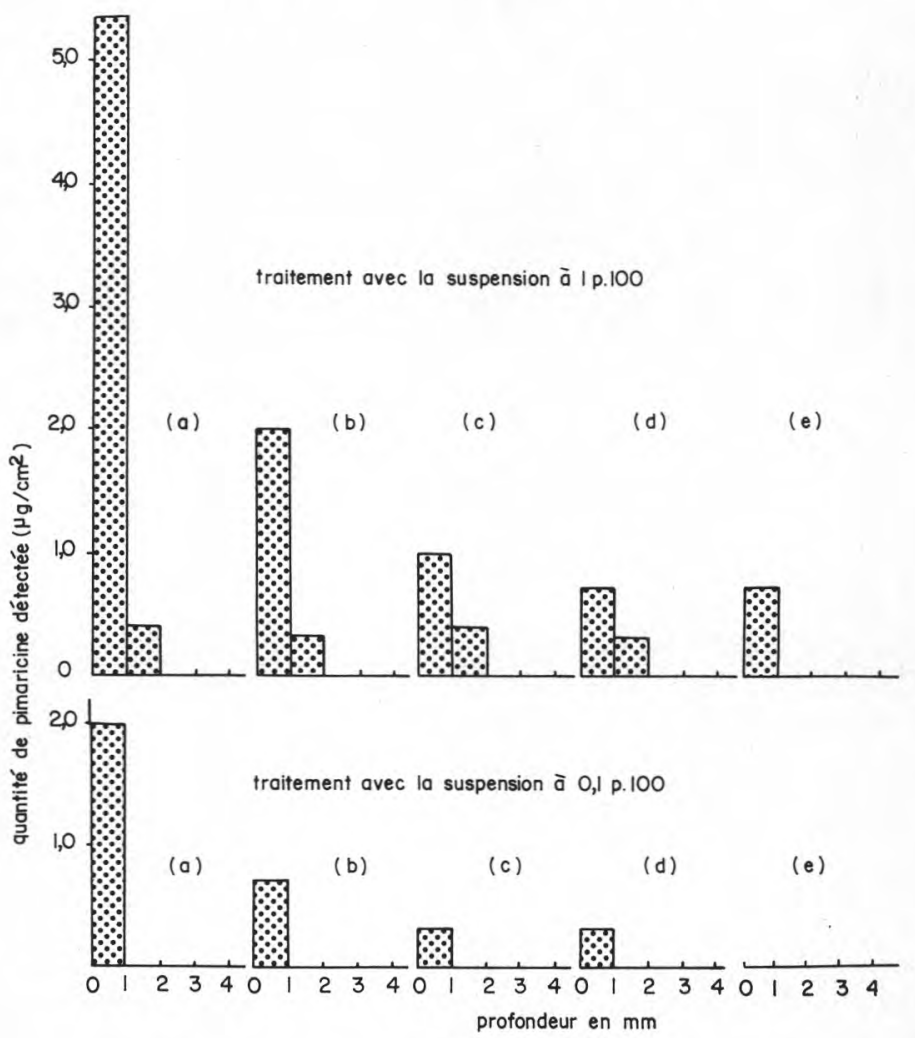

fig. 4

Pénétration de la pimaricine dans le "Bleu de Bresse " (Méthode des disques). Quantité de pimaricine détectée par $\mathrm{cm}^{2}$ à différentes profondeurs, $24 \mathrm{~h}$ (a), 1 semaine (b), 2 semaines (c), 3 semaines (d), 4 semaines (e) après traitement.

\section{3) Etude critique de la méthode de dosage par disques}

L'interprétation des résultats précédents est délicate car la méthode de dosage de la pimaricine par disques est susceptible d'un certain nombre de critiques.

a) Influence de la nature du caillé

La comparaison des quantités de pimaricine détectable (c'est-àdire des quantités de pimaricine qui ont diffusé de l'intérieur du disque dans la gélose) dans le premier disque en fonction de la face du disque qui est appliquée en contact avec la gélose a été faite sur le premier disque à partir de la surface (tab. 3 ). 
TABLEAU 3. - Quantité de pimaricine détectée dans le premier mm en fonction de la face du disque qui est appliquée sur la gélose ( $\mathrm{g} / \mathrm{cm}^{2}$ de surface de fromage)

\begin{tabular}{|c|c|c|c|c|c|c|c|c|c|c|c|c|}
\hline \multirow{3}{*}{$\begin{array}{l}\text { Date } \\
\text { de l'analyse } \\
\text { après le } \\
\text { traitement }\end{array}$} & \multicolumn{4}{|c|}{ "Pont l'Evêque » } & \multicolumn{4}{|c|}{ « Munster» } & \multicolumn{4}{|c|}{ «Bleu de Bresse» } \\
\hline & \multicolumn{2}{|c|}{0,1 p. 100} & \multicolumn{2}{|c|}{1 p. 100} & \multicolumn{2}{|c|}{0,1 p. 100} & \multicolumn{2}{|c|}{1 p. 100} & \multicolumn{2}{|c|}{0 , p. 100} & \multicolumn{2}{|c|}{1 p. 100} \\
\hline & $\begin{array}{l}\text { face } \\
\text { ext. }\end{array}$ & $\begin{array}{l}\text { face } \\
\text { int. }\end{array}$ & $\begin{array}{l}\text { face } \\
\text { ext. }\end{array}$ & $\begin{array}{l}\text { face } \\
\text { int. }\end{array}$ & $\begin{array}{l}\text { face } \\
\text { ext. }\end{array}$ & $\begin{array}{l}\text { face } \\
\text { int. }\end{array}$ & $\begin{array}{l}\text { face } \\
\text { ext. }\end{array}$ & $\begin{array}{l}\text { face } \\
\text { int. }\end{array}$ & $\begin{array}{l}\text { face } \\
\text { ext. }\end{array}$ & $\begin{array}{l}\text { face } \\
\text { int. }\end{array}$ & $\begin{array}{l}\text { face } \\
\text { ext. }\end{array}$ & $\begin{array}{l}\text { face } \\
\text { int. }\end{array}$ \\
\hline $24 \mathrm{~h}$ & 2,0 & 2,0 & 5,2 & 4,4 & 2,4 & 1,2 & 3,0 & 3,0 & 2,0 & 0,4 & 5,4 & 1,6 \\
\hline 1 semaine & 0,7 & 0,4 & 1,6 & 1,3 & 0,5 & 0,3 & 2,4 & 2,0 & 0,7 & 0,2 & 2,1 & 1,2 \\
\hline 2 semaines & 0,3 & 0,2 & 1,2 & 1,0 & 0,3 & 0,3 & 0,7 & 0,8 & 0,3 & $<0,3$ & 1,0 & 0,5 \\
\hline 3 semaines & 0,3 & $<0,3$ & 0,7 & 0,8 & 0,2 & 0,2 & 0,7 & 0,8 & 0,3 & $<0,3$ & 0,7 & 0,3 \\
\hline 4 semaines & 0,3 & $<0,3$ & 0,6 & 0,6 & 0,2 & 0,2 & 0,6 & 0,7 & 0,3 & $<0,3$ & 0,7 & $<0,3$ \\
\hline
\end{tabular}


Dans le cas des "Pont l'Evêque " et des " Munster " les quantités détectées sont sensiblement les mêmes quelle cue soit la face du disque qui est appliquée sur la gélose. Au contraire pour le "Bleu de Bresse » les quantités détectées lorsque la face inférieure du disque est appliquée sur la gélose sont systématiquement inférieures aux quantité 3 détectées lorsque la face supérieure du disque est appliquée sur la gélose. La pimaricine détectable ne diffuse donc pas librement de l'intérieur du disque vers la gélose et les quantités retrouvées ne sont pas représentatives des quantités de pimaricine « potentiellement détectables » présentes. Donc surtout dans le cas du «Bleu de Bresse » le dosage de la pimaricine par la méthode des disques ne peut être considéré comme quantitatif.

\section{b) Influence de la concentration en pimaricine}

Vingt-quatre heures après traitement avec la suspension de pimaricine à 0,1 p. 100 la pimaricine n'est détectée que dans le premier disque (fig. 2, 3 et 4). La valeur trouvée représente respectivement 60 et 40 p. 100 de la valeur initiale pour le "Pont l'Evêque » et le «Munster ». Dans le cas du traitement avec les suspensions à 1 p. 100 on ne peut connaître avec précision la quantité de pimaricine détectable dans le fromage qu'à partir de la $1^{\text {re }}$ semaine d'affinage lorsque les diamètres des zones d'inhibition sont dans la partie linéaire de la courbe étalon. La somme des quantités de pimaricine détectée dans chaque disque pour les "Munster " et les "Pont l'Evêque " ne représente que 5 à 10 p. 100 de la quantité initiale.

La quantité de pimaricine détectable ne représente donc qu'une certaine proportion de la quantité présente dans le fromage et cette proportion diminue lorsque la quantité de pimaricine appliquée sur le fromage augmente. Deux explications peuvent être trouvées à ce phénomène. On peut supposer que la fraction manquante a diffusé rapidement dans le caillé au point que dans les "Pont l'Evêque " et les "Munster " traités avec la dose de 1 p. 100 elle ne peut être dosée à partir du $5^{\mathrm{me}} \mathrm{mm}$. Il faudrait admettre que cette diffusion n'a lieu que pendant la $1^{\text {re }}$ semaine, puisque pour le "Munster " la quantité totale de pimaricine retrouvée varie entre la $1^{\text {re }}$ et la $4^{\text {me }}$ semaine. Une autre explication serait qu'à partir de certaines concentrations la pimaricine resterait bloquée dans le caillé par exemple sous forme de précipité, empêchant une diffusion rapide de la pimaricine dans la gélose et provoquant ainsi une réduction du diamètre de la zone d'inhibition. La proportion de pimaricine fixée augmenterait avec la concentration de la dose appliquée. Dans ces conditions la méthode de dosage ne serait plus quantitative.

\section{c) Influence de la maturation}

Pour les fromages traités avec la suspension à 0,1 p. 100 de pimaricine les quantités de pimaricine décelables diminuent au cours de l'affinage. Les quantités détectées après 4 semaines dans les "Munster ", les « Pont l'Evêque » et les «Bleu de Bresse " représentent 
respectivement 15,10 et moins de 7 p. 100 des quantités détectées $24 \mathrm{~h}$ après le traitement. Ces observations pourraient laisser supposer soit une disparition de l'activité antifongique de la pimaricine, soit une diminution des concentrations locales en pimaricine totale par dilution dans la masse du fromage. Comme on l'a vu plus haut, ces deux hypothèses sont infirmées par les résultats des dosages par extraction effectués au cours de la $5^{\text {me }}$ semaine d'affinage.

La disparité croissante dans le temps entre les quantités détectées et les quantités appliquées est donc due, soit à une lente fixation de la pimaricine détectable sur le fromage, soit à une diffusion de la pimaricine détectable seule vers l'intérieur du fromage. Dans cette dernière hypothèse on devrait observer la formation d'un gradient de diffusion. Or les "Pont l'Evêque " et les "Bleu de Bresse " ne présentaient pas de gradient de diffusion ce qui est un argument en faveur d'une fixation ou adsorption progressive de la pimaricine sur le fromage au cours de la maturation.

\section{CONCLUSION}

Comme il vient d'être montré précédemment la méthode des disques ne permet pas de doser la totalité de la pimaricine présente dans le disque de fromage. La relation entre la quantité de pimaricine détectable et la quantité de pimaricine totale est fonction de la nature du caillé, de la concentration en pimaricine et de l'état de maturation du caillé. Les conclusions ne peuvent donc porter que sur la pimaricine détectable seulement (c'est-à-dire la pimaricine qui diffuse dans la gélose).

Pour les fromages traités avec la suspension à 1 p. 100 de pimaricine les pentes des gradients de concentration en pimaricine détectable varient suivant le type de fromage. Les pentes se classent dans l'ordre décroissant suivant : "Munster ", "Pont l'Evêque " et «Bleu de Bresse ». Les profondeurs maximales de détection se classent dans le même ordre. Ces données conduiraient à penser que la pimaricine détectable diffuse plus facilement dans le "Munster », moins bien dans le "Pont l'Evêque » et encore moins bien dans le "Bleu de Bresse ».

Pour les fromages traités avec la dose de 0,1 p. 100 les gradients de concentration sont difficiles à mettre en évidence pour le "Pont l'Evêque » et le «Bleu de Bresse ». On peut toutefois dire que la pénétration de la pimaricine est au moins égale à la profondeur maximale de détection, soit $4 \mathrm{~mm}$ pour le « Munster », $2 \mathrm{~mm}$ pour le «Pont l'Evêque » et $1 \mathrm{~mm}$ pour le «Bleu de Bresse ».

En ce qui concerne le caillé «Bleu de Bresse » surtout, on a vu que le dosage par la méthode des disques ne pouvait être quantitatif quelle que soit la concentration de la suspension de pimari- 
cine utilisée pour le traitement, puisque la pimaricine ne diffuse pas complètement du disque dans la gélose. La faible pénétration de la pimaricine dans le «Bleu de Bresse " peut n'être qu'apparente par suite de la carence de la méthode de dosage. Inversement on peut tirer argument des observations au niveau de la méthode de dosage et au niveau de la pénétration dans le caillé pour conclure que le "Bleu de Bresse " représente le cas le plus marquant de la rétention de la pimaricine par le caillé, ce phénomène existant également à un degré moindre dans les deux autres types de caillé.

\section{Rés u mé}

La stabilité et la pénétration de la pimaricine ont été étudiées sur trois types de fromages à pâte molle préparés au laboratoire. Un fromage à coagulation lactique type "Munster ", un fromage à coagulation présure type "Pont l'Evêque » et un fromage type "Bleu de Bresse ». Ces fromages ont été traités avec des suspensions de pimaricine à 5,1 et $0,1 \mathrm{p}$. 100. La pimaricine a été dosée par une méthode de diffusion en gélose, soit à partir d'extrait méthanolique de fromage, soit directement à partir de disques découpés dans le fromage.

La pimaricine s'est révélée stable dans les fromages traités avec de fortes ( 5 p. 100$)$ et de faibles $(0,1$ p. 100) doses. Dans ce dernier cas la pimaricine est encore détectable après 4 semaines d'affinage.

La pénétration de la pimaricine a été suivie avec la méthode des disques de fromage. La pénétration est plus importante dans les fromages traités avec la dose à 1 p. 100 que dans ceux traités avec la dose de 0,1 p. 100 , avec des différences d'un type de fromage à l'autre. Dans le cas de la dose à 0,1 p. 100 la pimaricine est détectée à une profondeur de $1 \mathrm{~mm}$ dans les "Bleu de Bresse », $2 \mathrm{~mm}$ dans les "Pont l'Evêque " et $4 \mathrm{~mm}$ dans les « Munster ».

Des critiques peuvent être formulées vis-à-vis des méthodes de dosage de la pimaricine. Il apparaît que l'extraction de la pimaricine par le méthanol n'est pas complète dans les fromages traités avec de fortes doses.

Pour la méthode des disques de fromage la relation entre la quantité de pimaricine détectée et la quantité présente est fonction de la nature du caillé, de la concentration en pimaricine et du degré d'affinage du fromage.

\section{S u m m a r y}

Stability and penetration of pimaricin into soft cheeses

The stability and penetration of pimaricin were studied on 3 types ( Munster », "Pont l'Evêque » and «Bleu de Bresse ») of 
soft cheese which were made in laboratory. The cheeses were treated with suspensions in water containing 5,1 and $0.1 \mathrm{p} .100$ pimaricin. Pimaricin was assayed by diffusion in agar, using either methanolic cheese extracts or discs which were cutted out from the treated cheeses.

Pimaricin was stable in the cheeses treated with high (5 p. 100) and low $(0.1 \mathrm{p} .100)$ doses. In this last case pimaricin was still detectable after four weeks of ripening. The penetration of pimaricin was followed by the cheeses discs assay. Penetration is more important into the cheeses treated with 1 p. 100 pimaricin that in those treated with 0.1 p. 100 pimaricin. Differences in pimaricin penetration were observed according to the type of cheese : after treatment by 0.1 p. 100 pimaricin, pimaricin was detected at $1 \mathrm{~mm}$ depth in "Bleu de Bresse ", $2 \mathrm{~mm}$ in "Pont l'Evêque » and $4 \mathrm{~mm}$ in "Munster ».

Some criticisms could be formulated about the pimaricin assay. Pimaricin extraction by methanol was not complete from the cheeses treated with high doses. In the cheese discs assay, the relation between the amount of pimaricin present in the cheese and the amount detected was depending on the type of cheese, the pimaricin concentration and the ripening stage of the cheese.

\section{Remerciements}

Nous remercions les Laboratoires Standa qui ont bien voulu nous fournir la pimaricine nécessaire à cette étude ainsi que le Dr R.P. Struyk qui nous a conseillé pour les méthodes de dosage.

\section{Bibliographie}

ClARK (W. L.), SHIRK (R. J.) KLINE (E. F.) (1964). - Pimaricin a new fungistat. Microbial Inhibitors in Food.

MoL (J. J.) (1966). - A new type of fungicide for the prevention of mould growth on cheese rind. Neth Milk Dairy J., 20, 73.

Struyk (A.P.), Hoette (I.), Drost (G.), Waisvisz (J. M.), VAN EeK (T.) and HoogerHEIDE (J.C.) (1957-1958). - Pimaricin, a new antifungal antibiotic. Antibiotics Annual.

Von Lorch (L.), Negri (R.), Del Vecchio (R.), Stacchini (A.) (1968). - La pimaricina caratteristische e suo impiego nella prevenzione delle micotossicosi di origine alimentare. Revista Igiene e Sanita Pubblica, 24, 1. 